\title{
A. Im Internet verfügbare statistische Daten
}

Tanja Guggenbühl, Gérard Perroulaz and Xavier Tschumi Canosa

\section{OpenEdition}

\section{Journals}

Electronic version

URL: http://journals.openedition.org/sjep/126

DOI: $10.4000 /$ sjep.126

ISSN: 1663-9677

\section{Publisher}

Institut de hautes études internationales et du développement

\section{Printed version}

Date of publication: 1 avril 2007

Number of pages: $209-220$

ISBN: 978-2-88247-067-6

ISSN: $1660-5926$

\section{Electronic reference}

Tanja Guggenbühl, Gérard Perroulaz und Xavier Tschumi Canosa, «A. Im Internet verfügbare statistische Daten », Schweizerisches Jahrbuch für Entwicklungspolitik [Online], 26-1 | 2007, Online erschienen am: 21 Juni 2010, abgerufen am 08 September 2020. URL : http:// journals.openedition.org/sjep/126; DOI : https://doi.org/10.4000/sjep.126 


\section{A. Im Internet verfügbare statistische Daten}

\section{A.1. Handelsströme}

\section{A.1.1. Daten der Eidgenössischen Zollverwaltung (EZV)}

Die Eidgenössische Zollverwaltung (EZV) verfügt über ein Datenbankensystem, aus dem die Aussenhandelsstatistik der Schweiz hervorging. Die entsprechende Website ist unter der Adresse <http://www.ezv.admin.ch> > Themen $>$ Aussenhandelsstatistik zugänglich.

\section{$\square$ Verfügbarkeit der Daten}

Eine Rubrik der obigen Internetseite, „Produkte“, stellt alle notwendigen Informationen bereit, um Zugang zum gesamten Datenmaterial über den Aussenhandel der Schweiz online (SWISS-impex) oder auf CD-ROM zu erhalten. Diese Produkte sind alle gegen Zahlung erhältlich, doch sind die Daten in bestimmten Schweizer Bibliotheken über den Katalog $\mathrm{RERO}^{1} \mathrm{zu}$ finden. Ansonsten können die CD-Rom unter der Rubrik „Bestellungen“ bestellt werden. Die Papierversion dieser Statistik, die ebenfalls gegen Zahlung erhältlich war, wurde ab den Daten für 2006 aufgegeben.

Ein Auszug sämtlicher zum Aussenhandel der Schweiz verfügbaren Daten ist jedoch gratis unter den Rubriken „Gesamtübersicht“, „Länder“ und „Waren“ der oben genannten Internetseite erhältlich. Die Zahlen sind dort unter verschiedenen Punkten angeordnet und können meist am Bildschirm sichtbar gemacht und/oder im PDF- bzw. Excel-Format heruntergeladen werden.

Die nachstehenden Punkte führen diese Rubriken im Einzelnen auf.

\section{$\square$ Gesamtübersicht}

Diese Rubrik stellt die jährlichen Gesamtzahlen der Ein- und Ausfuhren sowie des Handelsbilanzsaldos der Schweiz seit 1980 bis zum letzten Jahr bereit, für das die Daten verfügbar sind. An dieser Stelle sind keine weiteren Einzelheiten nach Ländern oder Waren angegeben. Eine andere Tabelle der Rubrik führt die Zahlen zum Aussenhandel der Schweiz pro Einwohner für die gleichen Jahre auf.

Unter dieser Rubrik werden zudem die provisorischen monatlichen Gesamtzahlen der Ein- und Ausfuhren für das letzte vollständige Jahr und auch bis zum letzten abgelaufenen Monat aufgeführt. Im Allgemeinen sind die endgültigen Zahlen des Vorjahrs ab dem Monat Juni des laufenden Jahres verfügbar.

Bei all diesen Zahlen wird ein Unterschied gemacht zwischen dem Gesamtwert 1, ohne Edelmetalle, Schmucksteine, Kunstgegenstände und Antiquitäten, der hauptsächlich der Konjunkturbeobachtung dient, und dem Gesamtwert 2, der den Aussenhandel in seiner Gesamtheit umfasst.

$1<$ http://www.opac.rero.ch $>$. 


\section{$\square$ Länder}

Unter dieser Rubrik sind die Daten über die Ein- und Ausfuhren und den Handelsbilanzsaldo für die letzten vier Jahre geografisch nach Wirtschaftsräumen oder nach Kontinenten geordnet. Ferner sind dort die Daten zum Aussenhandel der Schweiz mit den wichtigsten Partnerländern verfügbar.

Die Daten nach Wirtschaftsräumen (Excel-Format) sind in grossen Gruppen zusammengefasst und liefern keine Angaben $\mathrm{zu}$ den einzelnen Ländern der Wirtschaftsräume. Für Angaben nach Ländern (Excel-Format) sind die Zahlen nach Kontinenten zu konsultieren.

Die Länder, aus denen sich die Wirtschaftsräume und die Kontinente zusammensetzen, sind in PDF-Dateien angelegt, die unter der Rubrik „Erläuterungen“ auf der Internetseite der Aussenhandelsstatistik (unter „Länderverzeichnis“") erhältlich sind.

Mh Hinweis: Die von der Eidgenössischen Zollverwaltung gehandhabte Einstufung der Länder in Wirtschaftsräume ist ihr eigen und muss somit aufmerksam geprüft werden vor einem Vergleich mit Klassifikationen aus anderen Quellen, insbesondere mit der vom Entwicklungshilfeausschuss der OECD erstellten DAC-Liste der Empfängerländer der öffentlichen Entwicklungshilfe. So sind beispielsweise die in den Daten der Eidgenössischen Zollverwaltung aufgeführten Aggregate der Entwicklungsländer und der Tranformationsländer keineswegs mit den Aggregaten der (am Schluss des Jahrbuchs abgedruckten) DAC-Liste vergleichbar.

Vermerk: Im gesamten Statistischen Teil des Jahrbuchs wird die Klassifikation der DAC-Liste der Empfängerländer der öffentlichen Entwicklungshilfe verwendet. Für Flüsse des Jahres 2004 oder früherer Jahre bestand diese Liste aus zwei Teilen, wobei der erste Teil die Entwicklungsländer und -gebiete und der zweite Teil die Transitionsländer und -gebiete umfasste. Für Flüsse ab dem Jahr 2005 besteht die Liste nur noch aus einem einzigen Teil, den Empfängerländern der öffentlichen Entwicklungshilfe.

\section{$\square$ Waren}

Unter dieser Rubrik sind die Ein- und Ausfuhren nach der Warenart geordnet (14 hauptsächliche Wirtschaftszweige ${ }^{2}$ ) oder nach dem Verwendungszweck (6 Warengruppen ${ }^{3}$, die zur Erstellung der Konjunkturprognosen und der Konten der volkswirtschaftlichen Gesamtrechnung verwendet werden). Die Nomenklaturen nach Warenart oder Verwendungszweck sind unter der Rubrik „Erläuterungen“ auf der Internetseite der Aussenhandelsstatistik (unter ,Warennomenklaturen") verfügbar.

Die Zahlen betreffen die letzten vier Jahre und sind im PDF-Format (zusammengefasst) oder im Excel-Format erhätlich.

2 Land- und Forstwirtschafts- und Fischereiprodukte; Energieträger; Textilien, Bekleidung und Schuhe; Papier und grafische Erzeugnisse; Leder, Kautschuk und Kunststoffe; Produkte der chemischen Industrie und damit verbundener Industrien; Steine und Erden; Metalle; Maschinen und Elektronik; Fahrzeuge, Präzisionsinstrumente, Uhren und Bijouterie; verschiedene übrige Waren; Edelmetalle und Schmucksteine; Kunstgegenstände und Antiquitäten.

3 Rohstoffe und Halbfabrikate; Energieträger; Investitionsgüter; Konsumgüter; Edelmetalle und Schmucksteine; Kunstgegenstände und Antiquitäten. 
Die Klassifikation der Ein- und Ausfuhren nach Warenart ist noch nach Ländern unterteilt, jedoch nur nach den wichtigsten Bezugs- oder Absatzländern der konsultierten Warengruppe. Dies ist bei der Klassifikation der Ein- und Ausfuhren nach dem Verwendungszweck der Waren nicht der Fall, wo nur ein allgemeiner Überblick gegeben wird.

Dieser Punkt bietet ferner eine Gesamtübersicht über die Ein- und Ausfuhren gemäss dem Internationalen Warenverzeichnis für den Aussenhandel (CTCI) ${ }^{4}$ Die SITC/CTCI ist eine von der Statistischen Kommission der Vereinten Nationen verwaltete Warennomenklatur, die anhand wirtschaftlicher Kriterien für die Erstellung von Aussenhandelsstatistiken aufgebaut wurde.

\section{$\square$ Analyse des Aussenhandels}

Diese Rubrik liefert zusätzliche Informationen zu den unter den vorhergehenden Punkten verfügbaren Angaben. Texte und Grafiken veranschaulichen die Entwicklung des Aussenhandels der Schweiz für das Jahr mit den letzten erhältlichen Daten gegenüber dem Vorjahr (oder den Vorjahren).

Die gleiche Rubrik stellt unter „Wirtschafträume“ eine Tabelle mit den 30 wichtigsten Handelspartnern der Schweiz für das Jahr mit den neuesten Angaben bereit.

\section{$\square$ Andere Angaben}

Weitere statistische Daten zum Aussenhandel der Schweiz werden unter anderen Rubriken auf der Internetseite der Eidgenössischen Zollverwaltung aufgeführt, wie die Ein- und Ausfuhren nach Transportmitteln oder nach Kantonen und Übertrittszonen.

Unter einer dieser Rubriken werden zudem die Aussenhandelsindizes für die letzten Jahre präsentiert. Unter einer anderen Rubrik werden insbesondere die schweizerischen Kriegsmaterialexporte für das Jahr vor dem laufenden Jahr aufgeführt (vgl. auch unten, Punkt A.1.3).

\section{A.1.2. Daten der Schweizerischen Nationalbank (SNB) ${ }^{5}$}

Die Schweizerische Nationalbank (SNB) gibt ebenfalls Daten über den Aussenhandel der Schweiz heraus, und zwar in ihrer Publikation Statistisches Monatsheft : <http://www.snb.ch> > Publikationen > Statistisches Monatsheft.

Kapitel I (Buchstabe I) liefert die Aussenhandelszahlen nach Verwendungszweck der Waren, nach Warenart und nach Ländern. Die Daten sind im PDFFormat (so wie sie in der Publikation erscheinen) sowie im Daten-Format (Excel oder Text) verfügbar. Die Publikation führt die jährlichen Gesamtzahlen der Einfuhren, der Ausfuhren und des Handelsbilanzsaldos auf.

Einschränkung: Im Datenformat müssen die monatlichen Zahlen manuell addiert werden, um die Gesamtzahlen pro Jahr zu erhalten.

4 Siehe: $<$ http://unstats.un.org > > Statistical Databases $>$ Classifications Registry $>$ Quicklinks $>$ SITC Rev.4 (Englisch).

5 Anmerkung der Redaktion: Kurz vor Druckgabe des Jahrbuchs wurde die Website der SNB umgestaltet: siehe Medienmitteilung der SNB vom 2. April 2007 „Die SNB mit neuer Website“. Wir werden im nächsten Jahr die Veränderungen im Einzelnen berücksichtigen. 
Im Unterschied zu den Zahlen der Eidgenössischen Zollverwaltung, welche die Aussenhandelsdaten von Jahr zu Jahr (Mitte des Jahres für das Vorjahr) bereitstellt, stellt die Schweizerische Nationalbank die Daten von Monat zu Monat (mit einer Frist von drei Monaten - z.B. im Februar für die Novemberdaten des Vorjahrs) bereit. Die Zahlen der Schweizerischen Nationalbank entsprechen dem Gesamtwert 1 der Eidgenössischen Zollverwaltung (d.h. ohne Edelmetalle, Schmucksteine, Kunstgegenstände und Antiquitäten).

Die Zahlen der SNB liefern keine Einzelheiten nach Ländern zum Aussenhandel der Schweiz mit den Entwicklungsländern, ausser für Indien.

MY Hinweis: Die Liste der Entwicklungsländer der Nationalbank ist nicht die gleiche wie jene, die in Teil B und C der vorliegenden Statistiken des Jahrbuchs verwendet wird. Dort wird die vom Entwicklungshilfeausschuss der OECD erstellte (am Schluss des Jahrbuchs abgedruckte) DAC-Liste der Empfängerländer der öffentlichen Entwicklunghilfe verwendet.

\section{A.1.3. Daten des Staatssekretariats für Wirtschaft (SECO)}

Das Staatssekretariat für Wirtschaft (SECO) stellt auf seiner Website Informationen nach Ländern betreffend die Aussenwirtschaftsbeziehungen der Schweiz bereit : <http://www.seco-admin.ch > >D (Deutsch) >Aussenwirtschaft >Länderinformationen. Bei den Länderinformationen handelt es sich um Dokumente im Word-Format, die regelmässig aktualisiert werden und Daten über das betreffende Land enthalten, welche über den Rahmen des Aussenhandels der Schweiz hinausgehen (z.B. geopolitische Daten, diplomatische Beziehungen zwischen der Schweiz und dem Land).

Ein Dokument mit dem Titel Quellenliste „Bilaterale Wirtschaftsbeziehungen“ kann von dieser Internetseite des SECO heruntergeladen werden. Es liefert zusätzliche Informationen zu den Länderblättern in Form von unentgeltlich zugänglichen Internet-Links und mit Hinweis auf Wirtschaftsdaten nach Ländern.

Ferner werden auf der Website des SECO Informationen über die Exportkontrollen, insbesondere betreffend Kriegsmaterial bereitgestellt, und zwar auf der Seite <http://www.seco-admin.ch> > Themen > Exportkontrollen >Kriegsmaterial. Auf dieser Seite werden im Teil „News“ die Zahlen für die Kriegsmaterialexporte nach Bestimmungsländern ab dem Monat Februar für das Vorjahr aufgeführt. Die Zahlen für frühere Jahre sind unter dem Link „Frühere Statistiken“ zu finden.

\section{A.2. Finanzflüsse}

\section{A.2.1. Daten der Schweizerischen Nationalbank (SNB) ${ }^{6}$}

Die Website der Schweizerischen Nationalbank (SNB) publiziert die vollständigsten Angaben über die Finanzflüsse aus und nach der Schweiz: <http:// www.snb.ch $>>$ Statistiken. Die Rubrik ,Statistiken“ versammelt neu alle statistischen Publikationen der Nationalbank, eine Übersicht findet sich auf der Seite „Statistische Publikationen“. Die Liste der Publikationen erscheint auf

6 Siehe Fussnote 5. 
der folgenden Seite der Website der BNS unter: <http://www.snb.ch $>>$ Publikationen.

\section{$\square$,Statistisches Monatsheft“}

Beim Anklicken des Links <http://www.snb.ch> >Publikationen > Statistisches Monatsheft - wird lediglich das neueste Statistische Monatsheft präsentiert; jedoch ermöglicht ein weiterer Link ganz unten auf der Seite den Zugang zu den seit dem Jahr 2000 archivierten Monatsheften. Alle Zahlen sind im PDFFormat, aber auch im Datenformat (Excel oder Text) erhältlich.

Kapitel F des Statistischen Monatshefts - Kapitalmarkt - führt die Zahlen betreffend die Anleihen ausländischer Schuldner in der Schweiz, öffentlich oder nicht öffentlich aufgelegt, nach Anleihenskategorien und nach Ländergruppen auf.

Kapitel S des Statistischen Monatshefts - Direktinvestitionen - führt die Zahlen betreffend die Direktinvestitionen im Ausland auf. Die jährlichen Investitionsflüsse (Kapitalexporte), der Kapitalbestand (Stand am Jahresende) sowie der Personalbestand in den schweizerischen Unternehmen im Ausland sind nach Ländern oder nach Branchen und Sektoren verfügbar.

Min Heis : Die Ländereinstufung der Schweizerischen Nationalbank ist ihr eigen und darf nicht mit den Klassifikationen anderer Quellen, insbesondere mit der vom Entwicklungshilfeausschuss der OECD erstellten DAC-Liste der Empfängerländer der öffentlichen Enwicklungshilfe verglichen werden. Einige Länder, die von der SNB der Gruppe der Schwellenländer zugeordnet werden, werden zum Beispiel vom DAC als Entwicklungsländer eingestuft.

Vermerk: Im gesamten Statistischen Teil des Jahrbuchs wird die Klassifikation der vom DAC erstellten Liste der Hilfeempfängerländer verwendet. Für Flüsse des Jahres 2004 oder früherer Jahre bestand diese Liste aus zwei Teilen, wobei der erste Teil die Entwicklungsländer und -gebiete und der zweite Teil die Transitionsländer und -gebiete umfasste. Für Flüsse ab dem Jahr 2005 besteht die Liste nur noch aus einem einzigen Teil, den Empfängerländern öffentlicher Entwicklungshilfe.

\section{$\square$,Entwicklung der Direktinvestitionen“}

Der Bericht Entwicklung der Direktinvestitionen erscheint jährlich als Beilage zur Dezemberausgabe der Publikation Statistisches Monatsheft (siehe oben) und ist unter <http://www.snb.ch> > Statistiken > Direktinvestitionen > Entwicklung der Direktinvestitionen erhältlich. Durch Anklicken dieses Links auf der Website der SNB steht die Publikation ab dem Jahr 2000 bis zum letzten erhältlichen Jahr zur Verfügung (im Dezember 2006 wurde der Bericht Entwicklung der Direktinvestitionen im Jahr 2005 veröffentlicht). Die Publikation enthält Tabellen und Kommentare zur Entwicklung der schweizerischen Direktinvestitionen im Ausland und der ausländischen Direktinvestitionen in der Schweiz für das Referenzjahr. 


\section{$\square$,Die Banken in der Schweiz“}

Diese jährlich im Sommer erscheinende Publikation ist durch Anklicken des entsprechenden Links unter der Rubrik „Publikationen“ auf der Website der SNB unter <http://www.snb.ch> > Publikationen > Die Banken in der Schweiz erhältlich. Die Ausgaben sind im PDF-Format ab 1996 (vollständige oder zusammengefasste Veröffentlichung) verfügbar, und nur die neueste Publikation bietet zusätzlich Zahlen im Excel- oder Text-Format.

Kapitel 1.5 der Publikation gibt die Aufteilung der Auslandsguthaben und -verpflichtungen in den Bilanzen von rund hundert Schweizer Banken nach Ländern an (Tabelle 32).

Definition: Die in der Bilanz einer Bank aufgeführten Geschäfte sind nur jene Geschäfte, die von der Bank auf eigene Rechnung getätigt werden.

Kapitel 2.2 der Publikation gibt die Aufteilung der Auslandsguthaben und -verpflichtungen in den Bilanzen derselben rund hundert Schweizer Banken nach Ländern, jedoch für Treuhandgeschäfte an (Tabelle 38).

Definition: Die in der Bilanz einer Bank aufgeführten Treuhandgeschäfte sind jene Geschäfte, die von der Bank auf Rechnung und Risiko ihrer Kunden getätigt werden.

Minweis: Dieses Kapitel umfasst nicht die Gesamtheit der Auslandsguthaben und -verpflichtungen, da nicht alle Banken dort aufgeführt sind und der bankähnliche Sektor (z.B. Vermögensverwalter, Treuhänder, Wechselstuben) nicht darin berücksichtigt ist. Ausserdem sind die Tabellen 32 und 38 der Publikation die einzigen Tabellen, die Zahlen nach Ländern aufführen.

\section{$\square$ Zahlungsbilanz und Auslandvermögen der Schweiz}

Unter der Rubrik der Publikationen der SNB : <http://www.snb.ch>>Publikationen >Zahlungsbilanz und Auslandvermögen der Schweiz erscheinen drei Beilagen zum Statistischen Monatsheft. Die eine ist der oben vorgestellte Bericht Entwicklung der Direktinvestitionen, die beiden anderen sind folgende:

- Die Beilage „Zahlungsbilanz der Schweiz“ erscheint jährlich ${ }^{6}$ und ist auf der Website der SNB im PDF-Format ab dem Jahr 1996 verfügbar. Der Bericht kommentiert die Entwicklung des Handels- und Kapitalsverkehrs der Schweiz mit dem Ausland im Referenzjahr (Güter- und Dienstleistungsströme, Kapitalströme) und enthält Tabellen mit Zahlen über einen Zeitraum von fünf Jahren.

ـ Die Beilage „Auslandvermögen der Schweiz“ ist für jedes Jahr im PDF-Format ab dem Jahr 2000 erhältlich ${ }^{7}$. Die Publikation erläutert die Entwicklung der Auslandsaktiven, der Auslandspassiven und des Nettovermögens der Schweiz, gemäss den Richtlinien des Internationalen Währungsfonds (IWF). Die Tabellen führen diese Zahlen ebenfalls über einen Zeitraum von fünf Jahren an.

6 Herausgabe Ende September eines Jahres zu den Zahlen des Vorjahrs.

Herausgabe Ende Dezember eines Jahres zu den Zahlen des Vorjahrs. 


\section{A.2.2. Daten des OECD-Entwicklungshilfeausschusses (DAC)}

Der Entwicklungshilfeausschuss der OECD (DAC) besitzt eine Website in englischer Sprache mit zahlreichen statistischen Daten: <http://www.oecd.org/ dac $>>$ Aid Statistics. Diese Website ist viel kompletter als diejenige in französischer Sprache (<http://www.oecd.org/cad>>Statistiques de l'aide). Besteht ein Dokument in der anderen Sprache, so wird dies mit einem Vermerk erwähnt, und ein Link führt zur Übersetzung hin.

\section{$\square$ Verfügbarkeit der Daten}

Das DAC stellt seine Daten in Form von kostenlos online zugänglichen Tabellen zur Verfügung, aber auch als CD-ROM (insbesondere die CD-ROM Statistiques sur le développement international, International Development Statistics CDROM), oder als Berichte in Papierform, die im Online-Buchladen der OECD ${ }^{8}$ gekauft oder in den Schweizer Bibliotheken über den Katalog RERO ${ }^{9}$ eingesehen werden können.

$\square$ Coopération pour le développement. Rapport 2006, Les Dossiers du CAD, Paris, OCDE, 2006.

$\square$ Statistischer Anhang zum Jahresbericht „Coopération pour le développement“

Der Statistische Anhang zum Bericht Coopération pour le développement (Statistical Annex of the Development Co-operation Report) stellt in rund vierzig Tabellen im Excel-Format eine vollständige Übersicht über die Finanzflüsse zwischen den Mitgliedsstaaten des DAC und den Hilfeempfängerländern bereit.

Das Inhaltsverzeichnis (Table of Contents) des Statistischen Anhangs ist zum Auffinden der gesuchten Tabellen nützlich.

Wh Hinweis: Die Tabellen des Statistischen Anhangs zum Jahresbericht Coo5 pération pour le développement sind nicht jedes Jahr gleich nummeriert, somit ist das Inhaltsverzeichnis massgebend.

Tabelle 13 des Anhangs zum Jahresbericht 2006 gibt die Finanzflüsse zwischen den Mitgliedsländern des DAC und den Hilfeempfängerländern für das Jahr 2005 in vier Kategorien an:

$\square$ Öffentliche Entwicklungshilfe (siehe unten, Punkt A.3.1.);

$\checkmark$ andere Beiträge des öffentlichen Sektors (die im Gegensatz zur öffentlichen Entwicklungshilfe zu Marktbedingungen gewährt werden);

๑ Schenkungen der privaten Organisationen;

๖ Privatkapital, das diesen Ländern zu marktwirtschaftlichen Bedingungen ausgezahlt wird (Direktinvestitionen, Exportkredite und Portfolioanlagen).

Tabelle 14 führt die gleichen Daten, jedoch für die Jahre 2002 bis 2005 sowie für den Durchschnitt der Jahre 1994-1995, d.h. zehn Jahre vor den letzten erhältlichen Daten auf.

Tabelle 37 gibt die durchschnittlichen jährlichen Wechselkurse (im Bericht 2006 für die Jahre 2001 bis 2005) gegenüber dem Dollar für die Devisen der

\footnotetext{
$<$ http://www.oecdbookshop.org >

$<$ http://www.opac.rero.ch>
} 
DAC-Länder an. Diese Angaben sind hilfreich bei Vergleichen mit den Zahlen anderer Quellen, die nicht in Dollar ausgedrückt sind.

Tabelle 38 führt das Bruttonationaleinkommen (BNE) und die Bevölkerungszahlen für jedes DAC-Mitgliedsland auf, was die Berechnung der verschiedenen Arten von Flüssen pro BNE-Einheit oder pro Einwohner ermöglicht (im Bericht 2006 für die Jahre 2003 bis 2005 sowie Durchschnitt der Jahre 1994 und 1995).

\section{$\square$ Online-Statistiken zur internationalen Entwicklung}

Die Website der statistischen Daten des DAC enthält einen Link zur Seite der Online-Statistiken über die internationale Entwicklung (International Development Statistics Online), was einen Zugang zu den Datenbanken des DAC (Datasets) über die Finanzflüsse in die Hilfeempfängerländer gewährt.

Seit Mai 2006 aggregiert ein neues System - OECD.Stat (sprich „OECD dot Stat"“) - diese Statistiken und bietet den Benutzern einen erleichterten Zugang zu den oben aufgeführten Kategorien von Finanzflüssen nach den Hilfeempfängerländern. Die Daten können nach Geberländern gesiebt und im Excel-Format exportiert werden. Ferner sind mit diesem System Daten über einen sehr langen Zeitraum (seit 1960) erhältlich.

\section{$\square$ Andere statistische Daten des DAC oder der OECD}

Das DAC stellt auf seiner Website auf der Seite mit den statistischen Daten auch Übersichtstafeln über die verschiedenen relevanten Flüsse oder Beträge für die Mitgliedsländer des DAC (Donor Aid Charts, Hilfe nach Gebern) sowie für die Hilfeempfängerländer (Recipient Aid Charts, Hilfe nach Empfängern) bereit.

Die DAC-Liste der Empfängerländer der öffentlichen Entwicklungshilfe (DAC List of ODA Recipients) ist ebenfalls auf dieser Internetseite verfügbar.

$Y$ Hinweis: Diese Liste wird regelmässig überarbeitet, und jedem Jahr an Finanzflüssen (namentlich die Flüsse öffentlicher Entwicklungshilfe) entspricht eine präzise, für das betreffende Jahr gültige Liste. Für Flüsse des Jahres 2004 oder früherer Jahre bestand diese Liste aus zwei Teilen, wobei der erste Teil die Entwicklungsländer und -gebiete und der zweite Teil die Transitionsländer und -gebiete umfasste. Für Flüsse ab dem Jahr 2005 besteht die Liste nur noch aus einem einzigen Teil, den Empfängerländern öffentlicher Entwicklungshilfe.

\section{A.2.3. Komponenten der für die Entwicklungsländer bestimmten langfristigen Finanzflüsse}

Die Weltbank präsentiert alljährlich in ihrer Publikation Global Development Finance $^{10}$ im Teil „Sources and Definitions“ ein Schema und eine Tabelle, die zum besseren Verständnis der Zusammensetzung der die Entwicklungsländer betreffenden Finanzflüsse beitragen. Das Schema führt die Komponenten der für die Entwicklungsländer bestimmten langfristigen Finanzflüsse auf. Die Tabelle situiert diese Finanzflüsse in der Gesamtheit der langfristigen Finanzflüsse, die in den verschiedenen Zwischenbilanzen der volkswirtschaftlichen

10 Jüngste Ausgabe: World Bank, Global Development Finance 2006: The Development Potential of Surging Capital Flows, II. Summary and Country Tables, Washington, 2006, S. xxv-xxvi. 
Gesamtrechung (Leistungsbilanz, Kapitalbilanz und Devisenbilanz) auftreten und sich in der Zahlungsbilanz ausgleichen.

[Dah Jahbuch 2006, Nr. 1, Statistiken, Teil A, Schema 2 und Tabelle 1, S. 227.

\section{A.3. Entwicklungshilfeflüsse}

\section{A.3.1. Daten des OECD-Entwicklungshilfeauschusses (DAC)}

Wie unter Punkt A.2.2. oben beschrieben, besitzt der Entwicklungshilfeauschuss der OECD (DAC) eine Website mit einem reichhaltigen statistischen Material, <http://www.oecd.org/dac> > Aid Statistics. Über die Verfügbarkeit der Daten des DAC wird ebenfalls im Abschnitt A.2.2. informiert.

\section{$\square$ Statistischer Anhang des Jahresberichts „Coopération pour le développement“}

Diese Publikation ist online auf Französisch oder auf Englisch (Statistical Annex of the Development Co-operation Report) erhältlich. Der Statistische Anhang bietet in rund vierzig Tabellen im Excel-Format eine Gesamtübersicht über die Finanzflüsse zwischen den Mitgliedsländern des DAC und den Hilfeempfängerländern.

Das Inhaltsverzeichnis (Table of Contents) des Statistischen Anhangs ist zum Auffinden der gesuchten Tabellen nützlich.

Nh Hinweis: Da die Tabellen des Statistischen Anhangs des Jahresberichts Coopération pour le développement nicht jedes Jahr gleich nummeriert sind, ist das Inhaltsverzeichnis diesbezüglich massgebend.

Grafik 1 des Anhangs zum Jahresbericht 2006 gibt eine Übersicht über die Beiträge öffentlicher Entwicklungshilfe der DAC-Mitgliedsstaaten im Jahr 2005, im absoluten Wert und in Prozent des Bruttonationaleinkommens (BNE). Tabelle 1 führt die Zahlen dieser Beiträge für die Jahre 2004 und 2005 an.

Definition: Das DAC gibt eine Definition des Aggregats öffentlicher Entwicklungshilfe, die in den technischen Anmerkungen am Schluss der Jahresberichte über die Entwicklungszusammenarbeit zu finden ist. (Diese Berichte sind im Papierformat käuflich zu erwerben oder stehen in der Bibliothek zur Verfügung, sind aber nicht direkt online erhältlich). Die Definition lässt sich wie folgt zusammenfassen: Die öffentliche Entwicklungshilfe besteht aus Darlehen und Schenkungen, die vom öffentlichen Sektor der Mitgliedsländer des DAC den Ländern und Gebieten der DAC-Liste der Empfängerländer der öffentlichen Entwicklungshilfe zu günstigen finanziellen Bedingungen mit dem hauptsächlichen Ziel gewährt werden, die wirtschaftliche Entwicklung dieser Länder zu erleichtern und ihre Lebensbedingungen zu verbessern.

MM Hinweis : Die Angaben zum Aggregat öffentlicher Hilfe, die für die Transitionsländer und -gebiete bestimmt war, bestehen nicht mehr im Statistischen Anhang für die Flüsse ab dem Jahr 2005.

Tabelle 14 des Anhangs zum Jahresbericht 2006 gibt die Einzelheiten der Finanzflüsse des Aggregats „öffentliche Entwicklungshilfe“ für jedes Mitgliedsland des DAC und für die vier Jahre mit den neuesten Daten, insbesondere die Flüsse bilateraler öffentlicher Entwicklungshilfe (Darlehen und Schenkungen) 
und die Flüsse multilateraler öffentlicher Entwicklungshilfe an. Die Tabelle führt auch die privaten Flüsse der nichtstaatlichen Organisationen nach den Entwicklungsländern auf.

Für eine Aufteilung der Flüsse nach Empfängerländern der Hilfe sämtlicher DAC-Mitgliedsländer für die letzten fünf Jahre wird auf Tabelle 25 (öffentliche Entwicklungshilfe zugunsten der Entwicklungsländer) verwiesen.

Tabelle 32 führt die Liste der 15 wichtigsten Empfängerländer öffentlicher Entwicklungshilfe für jedes DAC-Mitgliedsland in Prozent der gesamten ausgezahlten öffentlichen Entwicklungshilfe (Bruttozahlungen) für 1984-85, 1994-95 und 2004-05 auf. Die Tabelle ermöglicht einen Vergleich der geografischen Fokussierung der Hilfe der einzelnen Geberländer im Zeitverlauf und zwischen den Mitgliedsländern des DAC. Ferner wird aus Tabelle 32 die Aufteilung der bilateralen öffentlichen Entwicklungshilfe zwischen den Ländergruppen der DAC-Liste der Empfängerländer der öffentlichen Entwicklungshilfe und nach geografischen Einheiten für jedes Mitgliedsland des DAC ersichtlich.

Definitionen: Eine Bruttozahlung entspricht dem Gesamtbetrag, der im Verlauf einer gegebenen Rechnungsperiode ausgezahlt wurde. Eine Nettozahlung entspricht der Bruttozahlung abzüglich aller während der gleichen Rechnungsperiode erfolgten Kapitalrückzahlungen oder Spendenrückerstattungen.

\section{A.3.2. Daten der Direktion für Entwicklung und Zusammenarbeit (DEZA)}

Die Direktion für Entwicklung und Zusammenarbeit (DEZA) gibt jedes Jahr statistische Daten über die Hilfe der Schweiz an die Entwicklungsländer heraus. Diese Daten werden sequentiell veröffentlicht, und die Zahlen sind zwischen den verschiedenen Quellen nicht unbedingt die gleichen (Aktualisierung provisorischer Zahlen).

\section{$\square$,Jahresbericht der internationalen Zusammenarbeit der Schweiz““}

Der Jahresbericht wird von der DEZA und vom Staatssekretariat für Wirtschaft (SECO) gemeinsam publiziert. Diese beiden Bundesämter sind für die Koordination und die Umsetzung der internationalen Zusammenarbeit der Schweiz zuständig. Der Bericht erscheint Mitte des dem Referenzjahr der Statistiken folgenden Jahres (im Juli 2006 kam der Jahresbericht 2005 mit provisorischen Zahlen für 2005 und aktualisierten Zahlen für 2004 heraus).

Mh Hinweis: Da es sich um eine Aktualisierung der Zahlen für 2004 handelt, sind diese nicht zwangläufig dieselben wie jene Zahlen, die im Jahresbericht 2004 ein Jahr zuvor veröffentlicht worden waren. Da die Zahlen für 2005 provisorisch sind, sind sie auch nicht unbedingt die gleichen wie die aktualisierten Zahlen, die einige Monate später in der Publikation Aide de la Suisse aux pays en développement (siehe unten) herausgegeben werden.

Der Bericht ist gratis im Internet (im PDF-Format) erhältlich und kann in Papierversion (als Broschüre) auf der Website der DEZA unter <http://www. deza.admin.ch > > Dokumentation bestellt werden.

Der erste - thematische - Teil des Berichts vermittelt einen Überblick über die Tätigkeit der Schweiz im Bereich der internationalen Zusammenarbeit im Jahr 2005. Der zweite - statistische - Teil führt die Zahlen der öffentlichen Entwick- 
lungshilfe der Schweiz für das Referenzjahr des Berichts und für das Vorjahr auf. Dieser Teil gibt unter anderem auch die Aufwendungen für die bilaterale Hilfe von DEZA und SECO nach Ländern, diejenigen der DEZA für die humanitäre Hilfe und nach Tätigkeitsbereichen sowie die Aufwendungen des SECO nach Instrumenten an.

\section{$\square$ „Entwicklungshilfe der Schweiz““}

Diese Publikation der DEZA (in zweisprachiger Fassung Französisch und Deutsch) ist gewöhnlich im Verlauf des Frühjahrs erhältlich und betrifft die Zahlen des vorletzten Jahres (so erscheint die Statistik 2005 im Frühjahr 2007). Diese Angaben sind derzeit nur in Papierform verfügbar, doch kann ein Exemplar der Broschüre kostenlos bei der DEZA ${ }^{11}$ bezogen werden. Die Publikation, die bis zur Herausgabe im Jahr 2006 den Titel Aide de la Suisse aux pays en développement et aux pays en transition trug, ist 2007 unter dem deutschen Titel Entwicklungshilfe der Schweiz. Statistiken 2005 erschienen. Sie umfasst aber auch die Hilfeflüsse für die Länder des Südens und Ostens, die nicht in der DAC-Liste der Empfängerländer der öffentlichen Entwicklungshilfe enthalten sind.

Mh Hinweis: Da diese Publikation mehrere Monate nach dem Jahresbericht der internationalen Zusammenarbeit der Schweiz (siehe oben) erscheint, sind die Zahlen dort aktualisiert und nicht mehr provisorisch. Sie können somit für das gemeinsame Referenzjahr unterschiedlich sein.

Die Publikation führt folgende Hilfeflüsse auf:

$\square$ Öffentliche und private Hilfe an die Entwicklungsländer: Überblick, Entwicklung, Finanzierung nach Finanzierungsquellen aufgeschlüsselt ;

$\square$ Multilaterale Beiträge im Rahmen der öffentlichen Entwicklungshilfe;

$\square$ Bilaterale Finanzflüsse nach den Empfängerländern öffentlicher Entwicklungshilfe und den anderen Ländern des Südens und Ostens : nach Kontinenten, nach Ländergruppen gemäss Klassifikation des DAC und nach Ländern aufgeschlüsselt. Diese Tabelle ist eine Kurzfassung der Tabelle von Teil B des vorliegenden Teils ,,Statistiken“.

$\checkmark$ Bilaterale Beiträge: öffentliche und private bilaterale Hilfe, nach Kontinenten und Ländern aufgeschlüsselt;

๖ Öffentliche Hilfe der Kantone an die Empfängerländer öffentlicher Entwicklungshilfe und die anderen Länder des Südens und Ostens, nach Kantonen aufgeschlüsselt;

口 Öffentliche Hilfe der Gemeinden an die Empfängerländer öffentlicher Entwicklungshilfe und die anderen Länder des Südens und Ostens, nach Kantonen und Gemeinden aufgeschlüsselt;

口 Private Hilfe der nichtstaatlichen Organisationen (NRO) an die Empfängerländer öffentlicher Entwicklungshilfe und die anderen Länder des Südens und Ostens, nach Organisationen und nach Hilfeempfängerländern aufgeschlüsselt. 
Im Anhang zur Broschüre finden sich folgende Dokumente: die DAC-Liste der Empfängerländer der öffentlichen Entwicklungshilfe vom 1. Januar 2005 sowie die Liste der anderen Länder des Ostens und Südens (frühere Transitionsländer), ferner ein Verzeichnis der im Entwicklungsbereich tätigen privaten Entwicklungsorganisationen (NRO) mit ihren Kontaktadressen. 